Review

\title{
Future Directions for Monitoring Treatment Responses in Breast Cancer
}

Matthew P. Shupe ${ }^{\circledR}$, Lindsey J. Graham ${ }^{1}$, Erika J. Schneble ${ }^{2}$, Frederick L. Flynt ${ }^{1}$, Michael N. Clemenshaw ${ }^{3}$, Aaron D. Kirkpatrick ${ }^{4}$, Alexander Stojadinovic ${ }^{5}$, George E. Peoples ${ }^{6}$, Nathan M. Shumway ${ }^{1}$

1. Department of Hematology/Oncology

2. Department of Surgery

3. Department of Nuclear Medicine

4. Department of Diagnostic Radiology

5. Department of Surgical Oncology, San Antonio Military Medical Center (SAMMC), Brooke Army Medical Center, 3551 Roger Brooke Dr., Ft. Sam Houston, TX 78234, USA

6. Department of Surgical Oncology, Walter Reid National Military Medical Center, 8901 Rockville Pike, Bethesda, MD 20889, USA

$\triangle$ Corresponding author: Matthew P. Shupe, Brooke Army Medical Center, Department of Hematology/Oncology, 3551 Roger Brooke Dr., Ft. Sam Houston, TX 78234. Office: 210-916-0751; Fax: 210-916-3366; Email: matthew.p.shupe.mil@mail.mil; mattshupe@gmail.com

( ) Ivyspring International Publisher. This is an open-access article distributed under the terms of the Creative Commons License (http://creativecommons.org/ licenses/by-nc-nd/3.0/). Reproduction is permitted for personal, noncommercial use, provided that the article is in whole, unmodified, and properly cited.

Received: 2013.06.29; Accepted: 2013.07.24; Published: 2014.01.05

\begin{abstract}
In the prior review, we outlined the current standard of care for monitoring treatment responses in breast cancer and discussed the many challenges associated with these strategies. We described the challenges faced in common clinical settings such as the adjuvant setting, neoadjuvant setting, and the metastatic setting. In this review, we will expand upon future directions meant to overcome several of these current challenges. We will also explore several new and promising methods under investigation to enhance how we monitor treatment responses in breast cancer. Furthermore, we will highlight several new technologies and techniques for monitoring breast cancer treatment in the adjuvant, neoadjuvant and metastatic setting.
\end{abstract}

Key words: breast cancer, treatment monitoring, future directions, adjuvant, neoadjuvant, metastatic

\section{INTRODUCTION}

A number of modalities are used to monitor treatment response in breast cancer patients treated in the adjuvant, neoadjuvant and metastatic clinical setting. In the previous review, we outlined the current standard of care for monitoring treatment responses in breast cancer and noted many challenges associated with the current monitoring capabilities. In this review, we discuss several exciting and promising new methods under investigation to improve how we follow response in breast cancer. As previously discussed, the prevalence of breast cancer in the United States is nearly three million [1]. Even more concerning is that in younger women (ages 25-39) there seems to be an increase in the incidence of distant metastatic disease at initial diagnosis [2]. For most patients with metastatic disease, this represents an incurable illness and poses several challenges for providers as we balance the toxicities of treatment with helping to provide improved quality of life and overall survival. Part of providing breast cancer patients with the best quality of life is to ensure safe, inexpensive, non-invasive and accurate testing for the monitoring of treatment progress. In this review, we explore new technologies and techniques for the monitoring of breast cancer treatment in the adjuvant, neoadjuvant and metastatic setting.

\section{MONITORING TREATMENT RESPONSE IN THE ADJUVANT SETTING}

\section{SURGICAL ASPECTS}

For years, women have chosen between total mastectomy (TM) with or without reconstruction 
versus breast conserving surgery (BCS) consisting of lumpectomy followed by radiation as their primary surgical approach to early staged breast cancer. Surgical goals include complete removal of the tumor with clear margins and lymph node staging. Assessment of the primary tumor size and regional lymph nodes defines the pathologic stage and together with tumor characteristics such as hormone receptor status and HER2 expression levels provide an estimate of the prognosis to inform systemic therapy decisions. Node status as well as margin assessment dictates the need for additional procedures to ensure optimization of surgical therapy. Dilemmas of surgical management include balancing decisions between good oncologic surgery and cosmetic outcomes. Future directions include the use of newer surgical techniques and the use of new technology to minimize the need for second surgical procedures and the consumption of time and resources. Additionally, these new technologies may help prevent wound complications that would delay recovery and/or initiation of adjuvant therapies.

\section{Margin Assessment}

Re-excision after breast conserving surgery (BCS) is an unfortunate reality of breast surgery, and is reported to be necessary up to $50 \%$ of the time $[3,4]$. Improving methods to assess surgical margins in real-time could limit surgery, improve cosmesis, and decrease the incidence of second procedures. Some sites use intraoperative frozen section analysis of lumpectomy margins, but this strategy is laborious, resource intensive and consumes valuable operative time. Other sites perform larger lumpectomies in an attempt to avoid second procedures, but this practice results in worse cosmetic outcomes. Despite most centers attempt to balance the extent of lumpectomy between oncologic adequacy and cosmesis, approximately $20-25 \%$ of women will require re-excision. There is a need for accurate and cost-effective methods of better margin assessment in order to decrease this percentage of patients requiring second procedures.

Several different approaches have been suggested with varying amounts of data to support them. These primarily fall into two categories: technique or technology. Some proposed intraoperative margin assessment modalities include: cavity margin (CM) assessment, use of MarginProbe technology, micro-computed tomography (micro-CT), and intraoperative specimen mammography.

Assessing CM, as opposed to lumpectomy margins (LM), has been suggested to be a rapid, accurate and safe oncologic approach for margin evaluation [5] with $\mathrm{CM}$ shave assessment significantly reducing need for re-excision $[6,7]$ as well as the improved diagnosis of multifocality [8]. The basic concept of CM assessment is to complete the lumpectomy and then provide shave biopsies of a six-sided cube (superior, inferior, lateral, medial, superficial, and deep). The assessment of the $\mathrm{CM}$ avoids the false positive margin results that come from the handling, inking, and grossing of the lumpectomy specimen. The advantage of CM as opposed to the technologies to follow is that it does not require any special equipment and does not add appreciable time to the procedure. Unfortunately, the result of the CM assessment is not known at the time of procedure completion leading to some re-excisions.

A goal of new technologies is to provide the surgeon with margin information immediately at the time of surgery in both an effective and efficient manner. Manual palpation alone is highly inaccurate while frozen sectioning or touch prep cytology is both time-consuming and imprecise [9]. MarginProbe, a recently developed device for real-time intraoperative margin assessment, has been shown to have high sensitivity and specificity in distinguishing between normal and cancer tissues [10]. The device is intended to be applied to a lumpectomy specimen to aid surgeon judgment if additional tissue needs to be re-excised. In a randomized, double-armed trial, utilization of MarginProbe for positive margin detection in BCS was shown to decrease rate of repeat operations by $56 \%$ [11].

Radiographic examination is commonly used intra-operatively to confirm excision of image detected breast lesion and/or markers. The goal of intraoperative two-dimensional specimen mammography is similar to other technologies - alert the surgeon to proceed with additional excision of a positive or potentially close margin at the time of surgery [12]. The benefit of this method includes easy reproducibility and rapid assessment particularly if necessary equipment is located within the operating room [13]. However, continued dilemmas include the variability of results in predicting margin status secondary to limitations in specimen orientation and architectural distortion of the specimen itself such as dense parenchyma $[14,15]$.

Another method for radiographic assessment, Micro-CT has been presented as a tool not only for allowing intraoperative analysis of tumor location in breast lumpectomy but in shaved margins, sentinel lymph nodes, and mastectomy specimens [16]. Advantages include high spatial resolution with the ability to distinguish breast masses from fibrous tissue and benign from malignant calcifications [17]. Limitations include prolonged scanning time for image acquisition and processing prohibiting true real time 
margin assessment. However, as micro-CT continues to evolve towards real-time margin assessment, this device as well the other previously discussed modalities will aid to avert some of the pitfalls of standard lumpectomy and lead to a lower rate of re-excision and/or requirement for completion mastectomy.

\section{Surgical Complication Avoidance}

Assessment and treatment of the superficial margin has become problematic especially with the shift toward skin-sparing mastectomies and immediate breast reconstruction. Positive superficial margins require re-excision which not only delays further treatment, but also risks exposure of implanted tissue expanders or prosthesis with the potential for postoperative infections. Production of thin skin flaps in attempts to avoid positive superficial margins risks skin necrosis which may lead to subsequent delays in initiation of needed adjuvant therapies. In addition to the strategies for better margin assessment described above, new techniques and/or technologies for flap viability will help alleviate some of the potential problems with skin-sparing procedures.

Past methodologies to evaluate tissue perfusion intraoperatively have included evaluation of a variety of devices including Doppler, tissue oximetry, and fluorescein. A new methodology using intraoperative laser angiography with indocyanine green, otherwise known as the SPY system, has been introduced for real-time assessment of tissue perfusion that can correlate with clinical outcomes and guide surgical decision making [18]. Limitations of the SPY Interfusion Assessment System include the surgeon's subjectivity intraoperatively. More recently quantitative data has recently been examined in the form of "relative" fluoresce of necrosis as quantitative values to augment clinical judgment of flap viability [19].

\section{Nodal Assessment}

Axillary lymph node status serves as an important prognostic indicator in the staging of breast cancer patients. The introduction of sentinel lymph node biopsy (SLNB) has provided surgeons with a less morbid alternative to axillary lymph node dissection (AxLND). SLNB has become the standard for initial axillary staging in clinically node-negative patients. Previously, a positive SLNB dictated a follow-on AxLND; however, the ACSOG-Z11 trial demonstrated no survival benefit for patients undergoing an AxLND compared to those who were randomized to forego this procedure [20]. As a result, women undergoing BCS who meet criteria of Z-11, are now able to avoid the morbidity of AxLND. The question then becomes whether AxLND can be avoided after mastectomy and a positive SLNB. Sev- eral retrospective studies have attempted to address this question. One institutional database review compared outcomes between TM and BCS patients with positive SLNB who did not receive AxLND and demonstrated excellent outcomes between both groups [21]. In examination of the Surveillance, Epidemiology and End Results database (1998-2004), the clinicopathologic and outcome data were examined comparing patients who underwent SLNB alone versus SLNB with AxLND without a statistically significant difference in OS between these two groups at 50 months follow-up [22]. Ultimately, this question will require a prospective, randomized trial to conclude the safety of foregoing AxLND after a mastectomy and positive SLNB.

\section{RADIOLOGY ASPECTS}

As outlined in the previous review, mammography is the mainstay of surveillance imaging following curative treatment of breast cancer [23], however, an emerging imaging modality is digital breast tomosynthesis (DBT). DBT acquires images in the same orientation as conventional digital mammography but can display images in a three-dimensional manner. Several clinical studies have confirmed DBT's ability to improve screening performance for asymptomatic women with increased cancer detection rates while lowering screening recall rates. Furthermore, several small studies have suggested that two-view DBT may prove to be an alternative to obtaining additional mammographic views in the diagnostic or symptomatic setting [24-26]. Although preliminary early clinical trials suggest DBT may surpass conventional mammography for surveillance in breast cancer patients, appropriate clinical trials are needed. Other emerging modalities to include contrast enhanced mammography and molecular imaging remain possible future adjunct modalities for surveillance [27].

\section{MEDICAL ONCOLOGY ASPECTS}

After completion of adjuvant therapy, follow-up care focuses on detecting recurrent disease in the hopes of improving long-term survival. Early detection does not always equate with improved survival and oncologists have struggled with the decision to use intensive surveillance methods versus waiting to treat based on clinical recurrence. When looking at an average risk screening population, lead time and length biases can make interpretation of new screening test results difficult. Very large studies are often needed to truly ascertain whether mortality is improved, which is the gold standard of any screening test. In the adjuvant setting, we are dealing with a different "screening" population that is at much 
higher risk of recurrence. One significant challenge has been the heterogeneity of breast cancer biologies. Length bias tends to underestimate the effectiveness of early detection tools when breast cancers that are highly aggressive recur. If we could estimate a woman's rate of progression should they recur, we may be able to better develop new screening tests. This is the true challenge. How do we personalize cancer care to best formulate an efficient surveillance plan for each individual patient after completion of adjuvant therapy? Further discussion of future directions for the early detection of recurrence is beyond the scope of this review and will be elaborated upon in future projects.

\section{NEOADJUVANT SETTING SURGICAL ASPECTS}

After neoadjuvant therapy, surgery is used to obtain local disease control and also to determine response to neoadjuvant therapy. Patients generally fall into three clinically-relevant categories: pathologic complete response ( $\mathrm{pCR})$, partial clinical and pathologic response, and no clinical and pathologic response or actual progression of disease while on therapy (nonresponders). Patients with pCR do exceptionally well [28] and should proceed with their planned consolidation treatment with radiation and hormonal therapy as indicated based on their preclinical stage, choice of surgery, and hormonal status, respectively. HER2-positive patients should complete up to one year of trastuzumab therapy. Nonresponders are at an extremely high risk for disease and in one recent trial looking at the use of taxane-based therapy and radiation in the neoadjuvant setting, the recurrence rate was $33 \%$ in nonresponders versus $13 \%$ in those who obtained a CR [29]. In the face of significant heterogeneity in breast cancer, the use of gene expression analysis has helped us better ascertain the predictive value of different chemotherapeutic agents [30]. As exciting as some of these advances are, the need for well designed clinical trials in the neoadjuvant setting are required to advance the field. The NSABP and other national research organizations are targeting this population of patients to determine the best treatment options for these women who do not achieve pCR after neoadjuvant therapy. It would seem that some additional adjuvant therapy, preferably with a low toxicity profile would be ideal such as novel hormonal agents or even immunotherapy including cancer vaccines. In the section below, we outline the current research efforts aimed at this population of patients.

\section{Incomplete pCR Clinical Trials}

A search of clinical trials.gov reveals three clinical trials currently examining treatment options for breast cancer patients with incomplete pCR after neoadjuvant therapy. These trials include evaluation of eribulin, carboplatin, and trastuzumab/emtansine as adjuvant therapy for incomplete $\mathrm{pCR}$ patients.

A phase 2 trial "A Trial of Eribulin in Patients Who Do Not Achieve Pathologic Complete Response (pCR) Following Neoadjuvant Chemotherapy" (NCT01401959) evaluates adjuvant therapy with eribulin (a novel microtubule inhibitor) in treatment of patient who fail to achieve pCR (+/- trastuzumab). In this single center, non-randomized study three cohorts will be evaluated: triple-negative, hormone-receptor-positive/HER2-negative, and HER2-positive. The primary endpoint is 2-year disease-free survival (DFS) with secondary outcomes including the tolerability and toxicity of this regimen.

A phase 3 trial, "Carboplatin as Adjuvant Chemotherapy in Triple Negative Breast Cancer" (NCT01752686), is designed to investigate the efficacy of carboplatin as adjuvant therapy for triple negative breast cancer patients with residual pathologic disease after neoadjuvant chemotherapy. There is evidence that suggests woman with triple negative breast cancer are phenotypical and molecularly similar to BRCA-1 associated breast cancers leading to research trying to exploit DNA repair defects with the use of platinum agents [31]. This single center, randomized study patients will evaluate two groups: carboplatin vs. observation. Primary endpoint is 3 year disease-free survival between the carboplatin and control populations with secondary outcomes including overall survival (OS), $\mathrm{pCR}$ rate, the percentage of patients who receive BCS, and number of adverse events.

Also a phase 3 trial, "A Study of Trastuzumab/Emtansine Versus Trastuzumab as Adjuvant Therapy in Patients with HER2-Positive Breast Cancer Who Have Residual Tumor in the Breast or Axillary Lymph Nodes Following Preoperative Therapy" (NCT01772472), evaluates trastuzumab/emtansine versus trastuzumab alone as adjuvant therapy who fail to achieve $\mathrm{pCR}$ after neoadjuvant therapy. Trastuzumab/emtansine is an antibody-drug conjugate that links the cytotoxic agent, mertansine (DM1) to trastuzumab and has been recently FDA-approved for use in the metastatic setting [32]. In this 2-arm, randomized, open-label study primary outcomes include invasive disease-free survival (IDFS). Secondary outcomes include disease-free survival, overall survival, distant recurrence-free interval, and incidence of adverse events including cardiac events. 


\section{RADIOLOGY ASPECTS}

Breast MRI shows strong potential to help assess early response to treatment and help stratify risk of recurrence for patients. Early assessment of therapy allows for appropriate changes in therapy and decreases any potential delay in surgery. A recent prospective multi-institutional study showed the greatest relative ability of breast MR to predict $\mathrm{pCR}$ was prior to the second cycle of chemotherapy. This was best achieved using tumor volume measurements rather than greatest diameter of the mass [33]. The utility of volumetric measurements demonstrates breast MR's ability to perform functional measurements. Similarly other early prospective multi-institutional studies are evaluating the ability of MR spectroscopy to evaluate choline content which may be able to predict tumor response within a day or two after the initial dose of chemotherapy $[33,34]$.

Another area of active investigation is the use of diffusion weighted MR imaging to evaluate response to neoadjuvant therapy. Diffusion weighted MR imaging shows similar accuracy to contrast enhanced MR for monitoring neoadjuvant chemotherapy, this may be of use for patients with impaired renal function [27]. The utility of diffusion weighted imaging for predicting response prior to initiation of chemotherapy is unclear at this time. Richard et al. demonstrated that pretreatment apparent diffusion coefficients (ADCs) from diffusion imaging could predict responders and non-responders to therapy when accounting for tumor subtypes (i.e. triple negative, HER2-enriched, luminal A, or luminal B) while other investigators showed no difference in pre-therapy ADC values for responders versus non-responders [27, 35]. Currently an American College of Radiology Imaging Networks (ACRIN) multi-institutional protocol is evaluating if changes in ADC values after each treatment cycle is predictive of pathologic complete response.

Research with the use of 18F-fluorodeoxyglucose (18FDG) PET/CT for evaluation of response to neoadjuvant therapy is ongoing. A challenge in assessing metabolic response or progressive disease of primary and metastatic disease is due to the various PET image derived uptake values (lean body corrected, maximum, peak and mean) that can be generated for each region of interest. Hatt et al. compared all of these indices to include tumor volume assessment values using metabolically active tumor volume (MATV) and total lesion glycolysis (TLG) to predict early response to neoadjuvant chemotherapy in breast cancer patients. They found the differences in baseline and follow-up 18FDG PET-CT scans of TLG was the best predictor of response ( $96 \%$ sensitivity, $92 \%$ specificity and 94\% accuracy) compared SUVmax (63\% sensitivity, 92\% specificity and $77 \%$ accuracy) particularly in patients with ER-positive/HER2-negative disease after 2 cycles of neoadjuvant chemotherapy [36]. Further investigation and standardization should be performed before widespread use in clinical practice.

\section{MEDICAL ONCOLOGY ASPECTS}

As described in the previous paper, utilizing trends in serum tumor markers such as CA 15-3 to monitor tumor response to neoadjuvant therapy has been investigated. This method, however, has not proven reliable and therefore has not changed clinical practice recommendations. With the introduction of genomic assays such as Oncotype DX (Genomic Health, Inc., CA) and MammaPrint (Agendia, Irvine, $\mathrm{CA}$ and Amsterdam, The Netherlands), future directions aim less at monitoring response and more at accurately predicting response. With this strategy, only patients who would benefit from neoadjuvant chemotherapy would be exposed to therapy toxicities. Likewise, those who would not benefit from neoadjuvant chemotherapy could proceed to surgery without delay. Discussion on predicting response is beyond the scope of this paper and will be discussed in a future publication.

\section{METASTATIC SETTING \\ SURGICAL ASPECTS}

Surgery has a very modest role in the metastatic setting of breast cancer. Occasionally loco-regional recurrences can be resected if this is the only site of disease which is infrequent or if the locally recurrent disease is symptomatic and can be removed with acceptable morbidity. Infrequently, breast cancer metastasis can be surgically addressed if all known disease can be removed safely. Under these rare circumstances, post surgical monitoring is standard physical exam and radiographic monitoring which will be addressed below.

\section{RADIOLOGY ASPECTS}

Much investigation is ongoing assessing 18FDG PET-CT utilization for response to therapy and clinical staging of breast cancer. Recognizing limitations of anatomic imaging assessment criteria such as RECIST 1.1 , investigation and development of additional response criteria have been developed using 18FDG PET-CT. In 2009, the Positron Emission tomography Response Criteria in Solid Tumors (PERCIST) was proposed. The concept of PERCIST is that cancer response assessed with 18FDG PET-CT is continuous and time dependent with 18FDG uptake decreasing over time with effective treatment. Metabolic assessment of 18FDG is measured using the semi-qualitative 
standard uptake value (SUV) with the PERCIST criteria recommending use of the lean body mass corrected value, SUL. All foci of increased 18FDG uptake are compared to background level measured in the right hepatic lobe. Lesions with the greatest 18FDG are measured (up to 5 lesions, maximum 2 lesions per organ) and summed to determine a comparison metabolic baseline. PERCIST recommends performing 18FDG PET-CT at least 10 days after chemotherapy. Response to therapy is assessed as a percent change in SULpeak of an individual lesion or sum of lesion SULs [33].

Table I: Positron Emission Tomography Response Criteria in Solid Tumors (PERCIST); modified from Wahl et al. [33]

\begin{tabular}{ll}
\hline Response Category & Criteria \\
\hline Complete metabolic & $\begin{array}{l}\text { Visual disappearance of all metabolically } \\
\text { active tumor }\end{array}$ \\
response & $>30 \%$ decrease in SUL peak (minimum \\
sponse & $\begin{array}{l}0.8 \text {-unit decrease) in lesion with greatest } \\
\text { uptake (not necessarily same lesion) }\end{array}$ \\
Progressive metabolic & $>30 \%$ increase in SUL peak; (minimum \\
disease & $\begin{array}{l}\text { D.8-unit increase) } \\
>75 \% \text { increase in total lesion glycolysis }\end{array}$ \\
& Confirmed new lesions \\
Stable metabolic disease & Does not meet other criteria
\end{tabular}

As described in the previous neoadjuvant section, there are many PET image-derived uptake values. One study evaluating the EORTC and PERCIST Response evaluations in metastatic colorectal cancer found that the mean lean body corrected value (SULmean) was less susceptible to test to retest variance [34]. Future investigations are needed to standardized metabolic assessment criteria.

Recognizing a void in the evaluation of bone metastasis in breast cancer patients, researchers at The University of Texas MD Anderson Cancer Center developed anatomic imaging assessment criteria in 2004. The MD Anderson (MDA) criteria defined response into the four standard categories of complete response, partial response, progressive disease and stable disease of previously nonmeasureable bone metastatic disease in patients with bone only metastatic disease [35]. The MDA criteria allow for bone lesions without soft tissue component to be considered measureable and also accounts for the sclerotic healing response to therapy to be evaluated [37]. Using the MDA criteria noted an improved differentiation of patient responders to chemotherapy from nonresponders with bone only metastasis which corresponded to progression-free survival [38].

Another newly proposed assessment criteria, RECIST-Breast (RECIST-B), decreased the clinical criteria for defining progressive disease to $\geq 10 \%$ from current recommend $\geq 20 \%$ of the largest measured lesional CT size dimension in RECIST 1.1. This change demonstrated a promising, statistically significant, increase in concordance with WHO criteria, increasing from $94 \%$ to $97 \%$. [39].

PET-CT also allows for possible molecular and breast cancer-specific receptor imaging other than just the assessment of tumor glucose metabolic uptake with 18FDG. Estrogen receptors expressed in breast cancer are routinely obtained in initial tumor marker assessment and are being investigated as a potential PET imaging agent. Fluorine-18 is tagged to estradiol forming 18F-fluoroestriol (18F-FES) and being investigated in clinical imaging trials. In a small study in 2008 , investigators found $94 \%$ agreement of 18 F-FES with biopsy immunohistochemistry with the additional benefit of a noninvasive assessment of total tumor burden to include additional sites of metastatic disease [40]. Another study which used 18F-FES to aid in the assessment of treatment in breast cancer patients determined an improved diagnostic understanding by the referring providers in $88 \%$ of imaged patients and led to a change in therapy in $48 \%$ of patients included in the study. However, the researchers in this study concluded there was poor detection of liver metastasis [41].

Further advances in specific receptor imaging in breast cancer and the emerging technology of combined PET and MR (PET/MRI) imaging will lead to patient specific imaging as well as individualized targeted receptor radioimmunohistochemistry therapies.

\section{MEDICAL ONCOLOGY ASPECTS}

In contrast to monitoring patients with early stage breast cancer, the goal for follow-up and surveillance of patients with metastatic breast cancer are to influence treatment interventions in order to improve length and quality of life. Metastatic breast cancer is a heterogeneous disease with median survival approximately 2 years. The response to therapy is also variable [42]. Large, randomized trials to help guide clinicians in the monitoring of treatment responses in metastatic breast cancer are lacking. In this section, we will explore future directions for enhancing our ability to monitor disease, with the hope of ultimately changing the natural history of breast cancer in the metastatic setting.

In the metastatic setting, CA $15-3$ or CA 27.29 and CEA are recommended by ASCO for therapy monitoring and surveillance but should not be used solely in decision-making for therapy changes [42]. We still rely on radiologic findings, clinical examination and history taking in order to determine how a patient is responding to therapy. One of the biggest 
challenges is the lack of quality data in the study of breast cancer tumor markers. Most studies have small patient numbers, are retrospective in design, commonly perform multiple analyses until a significant finding is found and fail to include an accurate description of how the patients in each arm were treated. In addition, lack of standardization for the ordering of tumor markers is lacking with some assays using different reagents, procedures and scoring schemas. In order to improve the evaluation and reliability of tumor marker measurements in clinical trials, the members of an ASCO panel developed the Tumor Marker Utility Grading System (TMUGS). Tumor markers are assigned a utility score ranging from " 0 " to " +++ ". Only those assigned " ++ " or " +++ " are recommended for clinical use during treatment and subsequent follow-up to monitor patients, including the metastatic setting [43]. Reporting of tumor marker studies has also been variable. The National Cancer Institute and the NCI-EORTC published "REporting recommendations for tumour MARKer prognostic studies (REMARK)" to encourage transparent and complete reporting of tumor marker studies [44]. Rigorous tumor marker trial design using TMUGS and complete reporting recommended by REMARK guidelines should lead the use of well-validated tumor markers for routine clinical use [45].

\section{Circulating Tumor Cells}

Perhaps, one of the most exciting areas of research revolves around the use of circulating tumor cells (CTCs). Breast cancer is staged on the basis of tumor size, lymph node status, and the presence or absence of metastatic disease. Currently, axillary lymph node status acts as the best predictor of outcomes in respect to operable breast cancer. However, $25 \%$ percent of patients without axillary lymph-node metastasis will undergo systemic relapse [46]. This paradox may be explained by the presence of micrometastatic disease as measured by circulating tumor cells (CTCs). This early hematogenous spread of tumor cells can occur in patients with operable breast cancer independent of lymphatic involvement.

Quantification of these CTCs in peripheral blood before initiation of systemic treatment has been shown to be predictive of progression-free and overall survival in metastatic breast cancer [47]. CellSearch is an FDA-approved detection system for identifying and quantifying CTC, and it has been approved for clinical use in the metastatic setting of breast cancer to guide response to therapy.

Attempts to extend the use of CTC to earlier staged breast cancer have led to the investigation of even more sensitive methods of detecting CTC. In general, CTC detection occurs through two steps: en- richment or isolation through immunological techniques followed by detection and identification with nucleic acid and/or cytometric methods. Various types of detection assays have been used to identify CTCs in stage 1-2 non-metastatic breast cancer to include cytokeratin-19mRNA amplification[48-50], cytokeratin immunostaining [51], HER2 immunostaining, and the CellSearch (Veridez, Raritan, NJ) method [52-55]. Specifically with the CellSearch method, CTCs have been identified in $31 \%$ of patients with $\mathrm{T} 1$ or T2 tumors suggesting occult dissemination to occur early in disease progression [56].

Identifying the presence of CTCs may act as an important prognostic variable to identify patients who could potentially benefit from additional adjuvant therapies creating a new treatment algorithm. Future directions include improving CTC analysis to predict clinical outcome with approaches of both high accuracy and reproducibility. Furthermore, characterization of CTCs offers researchers the possibility to dissect underlying mechanisms of metastasis and drug resistance.

\section{HER2 Extracellular Domain}

Another potential biomarker prompting numerous investigations for monitoring metastatic breast cancer is the extracellular domain (ECD) of HER2. The ECD of the HER2 protein can be cleaved from the extracellular surface of breast cancer cells by matrix metalloproteases where it can be detected in the serum by ELISA [57]. Several studies have investigated if serum HER2 ECD levels at baseline or during therapy with trastuzumab can be a potential marker for tumor response or disease progression. Kostler et al suggested that early changes in serum HER2 ECD could predict responses to trastuzumab therapy and progression-free survival in metastatic breast cancer [58]. Others have suggested that increasing levels of HER2 ECD could represent disease recurrence or progression. Most of these trials were relatively small prompting Lennon et al. to investigate in a retrospective analysis whether HER2 ECD measurements were useful for clinical decision-making. No significant relationship was found between initial change in serum ECD and overall tumor response and almost all patients with normal baseline ECD levels experienced no change or only a slight increase in levels prior to or at disease progression [57]. Although appealing, larger prospective studies are required before HER2 ECD can be recommended for clinical use in metastatic breast cancer [59].

\section{Novel Biomarkers}

Other novel biomarkers for monitoring metastatic breast cancer are under clinical investigation 
including circulating tumor DNA, hypermethylated gene promoters, and low molecular weight serum proteins using nanoporous silica chips. DNA fragments carrying individual, tumor specific alterations can be detected in serum allowing for personalized assays for monitoring metastatic breast cancer. A recent prospective, single-center study involving 30 women with metastatic breast cancer found improved sensitivity of circulating tumor DNA compared to CA $15-3$ (85\% vs. 59\%) and dynamic increases in levels prior to progressive disease. Circulating tumor DNA is an exciting, potential "liquid biopsy" alternative that provides innately specific and highly sensitive information during the course of metastatic breast cancer [60]. Circulating hypermethylated DNA biomarkers are another promising application for monitoring patients treated for metastatic breast cancer. Serum levels of methylated gene promoter 14-3-3-o (sratifin) can be quantitatively measured by methylation-specific PCR. Zurita et al found that serum levels of 14-3-3- $\sigma$ methylated gene promoter discriminated between healthy individuals and metastatic breast cancer with a sensitivity of $81 \%$ and specificity of $96 \%$, thus concluding that 14-3-3-o could be used in patients treated for metastatic breast cancer to monitor their disease status and treatment response [61]. Lastly, recent developments in nanoporous chip-based technologies have allowed for capture of low molecular weight (LMW) serum proteins for monitoring progression of metastatic breast cancer. Using mouse models of human breast cancer lung metastases, Fan et al found unique levels of expression of LMW proteins for different stages of breast cancer lung metastases; some as early as two weeks after inoculation [62]. New promising modalities such as circulating tumor DNA, hypermethylated gene promoters, and LMW protein detection are emerging that hopefully will revolutionize follow-up care for metastatic breast cancer.

\section{CONCLUSION}

There are several opportunities to improve how we monitor breast cancer treatment. Only by understanding the current standard of care regarding all aspects of treatment can we start to move the field forward in a meaningful way. Several fields of cancer care including surgery, radiation oncology, pathology, radiology and medical oncology need to work together to ensure proper study design and coordination of care. We sought to provide a broad overview of these different fields of cancer care and believe that future clinical trials will need to be performed in several of the different clinical pathways discussed to provide more granularity and an accelerated pace of discovery for breast cancer researchers. The current paradigm of first testing new techniques or technologies in the metastatic setting prior to implementation in the adjuvant setting has been challenged by researchers who wish to speed the progress in breast cancer research. We believe that several opportunities exist to enhance breast cancer care by performing research in the neo-adjuvant setting as outlined in the sections above.

To help us better design these new trials, several national cancer care organizations have made recommendations regarding appropriate clinical trial design, use of appropriate tumor markers utilizing the TMUGS and consistent reporting of studies according to the REMARK recommendations. While this review has discussed several noteworthy and exciting areas for improving breast cancer treatment monitoring, we need a push to engage the community cancer care centers. When the community starts to embrace these concepts and adopts them into their own clinical pathways, the pace of breast cancer care discoveries should quicken.

In summary, we are entering an exciting time in the field of breast cancer research and new surgical, radiological, serologic, and biologic discoveries are ensuring that we continually improve how we monitor breast cancer patients receiving therapy. We hope to someday have a personalized approach to cancer care that incorporates many of the discoveries outlined above.

\section{ABBREVIATIONS}

18FDG: 18F-fluorodeoxyglucose; 18F-FES: 18F-fluoroestriol; ACRIN: American College of Radiology Imaging Networks; ACSOG: American College of Surgeons Oncology Group; ADC: apparent diffusion coefficients; ASCO: American Society of Clinical Oncology; AxLND: axillary lymph node dissection; BCS: breast conserving surgery; BRCA: breast cancer gene; CA: carcinoma antigen; CEA: carcinoembryonic antigen; CM: cavity margin; CT: computed tomography; CTC: circulating tumor cells; DBT: digital breast tomosynthesis; DFS: disease-free survival; DM1: mertansine; DNA: deoxyribonucleic acid; ECD: extracellular domain; EORTC: European Organisation for Research and Treatment of Cancer; ER: estrogen receptor; FDA: Food and Drug Administration; HER2: human epidermal growth factor receptor 2; IDFS: invasive disease-free survival; LM: lumpectomy margin; LMW: low molecular weight; MATV: metabolically active tumor volume; MDA: MD Anderson; Micro-CT: micro-computed tomography; MR: magnetic resonance; MRI: magnetic resonance imaging; NSABP: National Surgical Adjuvant Breast and Bowel Project; OS: overall survival; pCR: pathologic complete response; PCR: polymerase chain reaction; 
PERCIST: Positron Emission tomography Response Criteria in Solid Tumors; PET: positron emission tomography; RECIST: Response Evaluation Criteria In Solid Tumors; REMARK: REporting recommendations for tumour MARKer; SLNB: sentinel lymph node biopsy; SPY: intraoperative laser angiography; SULmean: mean lean body corrected value; SUV: standardized uptake value; TLG: total lesion glycolysis; TM: total mastectomy; TMUGS: Tumor Marker Utility Grading System; WHO: World Health Organization.

\section{Disclaimer}

The opinions or assertions herein are the private views of the authors and not to be construed as reflecting the views of the Department of the Army, Department of the Air Force, or the Department of Defense.

\section{Competing Interests}

The authors have declared that no competing interest exists.

\section{References}

1. Siegel R, DeSantis C, Virgo K, Stein K, Mariotto A, Smith T, et al. Cancer treatment and survivorship statistics, 2012. CA Cancer J Clin. 2012; 62: 220-41. doi:10.3322/caac.21149.

2. Johnson $\mathrm{RH}$, Chien FL, Bleyer A. Incidence of breast cancer with distant involvement among women in the United States, 1976 to 2009. JAMA. 2013; 309: 800-5. doi:10.1001/jama.2013.776.

3. Menes TS, Tartter PI, Bleiweiss I, Godbold JH, Estabrook A, Smith SR. The consequence of multiple re-excisions to obtain clear lumpectomy margins in breast cancer patients. Annals of Surgical Oncology. 2005; 12: 881-5. doi:Doi 10.1245/Aso.2005.03.021.

4. Mullenix PS, Cuadrado DG, Steele SR, Martin MJ, See CS, Beitler AL, et al. Secondary operations are frequently required to complete the surgical phase of therapy in the era of breast conservation and sentinel lymph node biopsy. American Journal of Surgery. 2004; 187: 643-6. doi:DOI 10.1016/j.amjsurg.2004.01.003.

5. Chen $\mathrm{K}$, Zeng Y, Jia H, Jia W, Yang $\mathrm{H}$, Rao N, et al. Clinical outcomes of breast-conserving surgery in patients using a modified method for cavity margin assessment. Ann Surg Oncol. 2012; 19: 3386-94. doi:10.1245/s10434-012-2331-5.

6. Jacobson AF, Asad J, Boolbol SK, Osborne MP, Boachie-Adjei K, Feldman SM. Do additional shaved margins at the time of lumpectomy eliminate the need for re-excision? Am J Surg. 2008; 196: 556-8. doi:10.1016/j.amjsurg.2008.06.007.

7. Janes SE, Stankhe M, Singh S, Isgar B. Systematic cavity shaves reduces close margins and re-excision rates in breast conserving surgery. Breast. 2006; 15: 326-30. doi:10.1016/j.breast.2005.10.006.

8. Feron JG, Nguyen A, Bezu C, Antoine M, Darai E, Coutant C, et al. Interest in cavity shaving in breast conservative treatment does not depend on $\begin{array}{llll}\text { lumpectomy technique. } & \text { Breast. 2011; 20: 358-64. }\end{array}$ doi:10.1016/j.breast.2011.01.014.

9. Klimberg VS, Harms S, Korourian S. Assessing margin status. Surg Oncol. 1999; 8: 77-84. doi:S0960-7404(99)00031-6 [pii].

10. Pappo I, Spector R, Schindel A, Morgenstern S, Sandbank J, Leider LT, et al. Diagnostic performance of a novel device for real-time margin assessment in lumpectomy specimens. J Surg Res. 2010; 160: 277-81. doi:10.1016/j.jss.2009.02.025.

11. Allweis TM, Kaufman Z, Lelcuk S, Pappo I, Karni T, Schneebaum S, et al. A prospective, randomized, controlled, multicenter study of a real-time, intraoperative probe for positive margin detection in breast-conserving surgery. Am J Surg. 2008; 196: 483-9. doi:10.1016/j.amjsurg.2008.06.024.

12. Bathla L, Harris A, Davey M, Sharma P, Silva E. High resolution intra-operative two-dimensional specimen mammography and its impact on second operation for re-excision of positive margins at final pathology after breast conservation surgery. Am J Surg. 2011; 202: 387-94. doi:10.1016/j.amjsurg.2010.09.031.

13. Kaufman CS, Jacobson L, Bachman BA, Kaufman LB, Mahon C, Gambrell LJ, et al. Intraoperative digital specimen mammography: rapid, accurate results expedite surgery. Ann Surg Oncol. 2007; 14: 1478-85. doi:10.1245/s10434-006-9126-5.
14. Chagpar A, Yen T, Sahin A, Hunt KK, Whitman GJ, Ames FC, et al. Intraoperative margin assessment reduces reexcision rates in patients with ductal carcinoma in situ treated with breast-conserving surgery. Am J Surg. 2003; 186: 371-7. doi:S0002961003002642 [pii].

15. Graham RA, Homer MJ, Sigler CJ, Safaii H, Schmid CH, Marchant DJ, et al. The efficacy of specimen radiography in evaluating the surgical margins of impalpable breast carcinoma. AJR Am J Roentgenol. 1994; 162: 33-6. doi:10.2214/ajr.162.1.8273685

16. Tang R, Buckley JM, Fernandez L, Coopey S, Aftreth O, Michaelson J, et al. Micro-computed tomography (Micro-CT): a novel approach for intraoperative breast cancer specimen imaging. Breast Cancer Res Treat. 2013; 139: 311-6. doi:10.1007/s10549-013-2554-6.

17. Gufler H, Franke FE, Wagner S, Rau WS. Fine structure of breast tissue on micro computed tomography a feasibility study. Acad Radiol. 2011; 18: 230-4. doi:10.1016/j.acra.2010.10.006.

18. Gurtner GC, Jones GE, Neligan PC, Newman MI, Phillips BT, Sacks JM, et al. Intraoperative laser angiography using the SPY system: review of the literature and recommendations for use. Ann Surg Innov Res. 2013; 7: 1. doi:10.1186/1750-1164-7-1.

19. Newman MI, Jack MC, Samson MC. SPY-Q analysis toolkit values potentially predict mastectomy flap necrosis. Ann Plast Surg. 2013; 70: 595-8. doi:10.1097/SAP.0b013e3182650b4e.

20. Giuliano AE, Hunt KK, Ballman KV, Beitsch PD, Whitworth PW, Blumencranz PW, et al. Axillary dissection vs no axillary dissection in women with invasive breast cancer and sentinel node metastasis: a randomized clinical trial. JAMA. 2011; 305: 569-75. doi:10.1001/jama.2011.90.

21. Milgrom S, Cody H, Tan L, Morrow M, Pesce C, Setton J, et al. Characteristics and outcomes of sentinel node-positive breast cancer patients after total mastectomy without axillary-specific treatment. Ann Surg Oncol. 2012; 19: 3762-70. doi:10.1245/s10434-012-2386-3.

22. Yi M, Giordano SH, Meric-Bernstam F, Mittendorf EA, Kuerer HM, Hwang $\mathrm{RF}$, et al. Trends in and outcomes from sentinel lymph node biopsy (SLNB) alone vs. SLNB with axillary lymph node dissection for node-positive breast cancer patients: experience from the SEER database. Ann Surg Oncol. 2010; 17 Suppl 3: 343-51. doi:10.1245/s10434-010-1253-3.

23. Arasu VA, Joe BN, Lvoff NM, Leung JW, Brenner RJ, Flowers CI, et al. Benefit of semiannual ipsilateral mammographic surveillance following breast conservation therapy. Radiology. 2012; 264: 371-7. doi:10.1148/radiol.12111458

24. Hakim CM, Chough DM, Ganott MA, Sumkin JH, Zuley ML, Gur D. Digital breast tomosynthesis in the diagnostic environment: A subjective side-by-side review. AJR Am J Roentgenol. 2010; 195: W172-6. doi:10.2214/AJR.09.3244.

25. Noroozian M, Hadjiiski L, Rahnama-Moghadam S, Klein KA, Jeffries DO, Pinsky RW, et al. Digital breast tomosynthesis is comparable to mammographic spot views for mass characterization. Radiology. 2012; 262: 61-8. doi:10.1148/radiol.11101763.

26. Skaane P, Gullien R, Bjorndal H, Eben EB, Ekseth U, Haakenaasen U, et al. Digital breast tomosynthesis (DBT): initial experience in a clinical setting. Acta Radiol. 2012; 53: 524-9. doi:10.1258/ar.2012.120062.

27. Drukteinis JS, Mooney BP, Flowers CI, Gatenby RA. Beyond mammography: new frontiers in breast cancer screening. Am J Med. 2013; 126: 472-9. doi:10.1016/j.amjmed.2012.11.025.

28. Fisher B, Bryant J, Wolmark N, Mamounas E, Brown A, Fisher ER, et al. Effect of preoperative chemotherapy on the outcome of women with operable breast cancer. Journal of Clinical Oncology. 1998; 16: 2672-85.

29. Adams S, Chakravarthy AB, Donach M, Spicer D, Lymberis S, Singh B, et al. Preoperative concurrent paclitaxel-radiation in locally advanced breast cancer: pathologic response correlates with five-year overall survival. Breast Cancer Res Treat. 2010; 124: 723-32. doi:10.1007/s10549-010-1181-8.

30. Chen YZ, Xue JY, Chen CM, Yang BL, Xu QH, Wu F, et al. PPAR signaling pathway may be an important predictor of breast cancer response to neoadjuvant chemotherapy. Cancer Chemother Pharmacol. 2012; 70: 637-44. doi:10.1007/s00280-012-1949-0.

31. Sirohi B, Arnedos M, Popat S, Ashley S, Nerurkar A, Walsh G, et al. Platinum-based chemotherapy in triple-negative breast cancer. Ann Oncol. 2008; 19: 1847-52. doi:10.1093/annonc/mdn395.

32. Verma S, Miles D, Gianni L, Krop IE, Welslau M, Baselga J, et al. Trastuzumab emtansine for HER2-positive advanced breast cancer. N Engl J Med. 2012; 367: 1783-91. doi:10.1056/NEJMoa1209124.

33. Wahl RL, Jacene H, Kasamon Y, Lodge MA. From RECIST to PERCIST: Evolving Considerations for PET response criteria in solid tumors. J Nucl Med. 2009; 50 Suppl 1: 122S-50S. doi:10.2967/jnumed.108.057307.

34. Skougaard K, Nielsen D, Jensen BV, Hendel HW. Comparison of EORTC Criteria and PERCIST for PET/CT Response Evaluation of Patients with Metastatic Colorectal Cancer Treated with Irinotecan and Cetuximab. J Nucl Med. 2013. doi:10.2967/jnumed.112.111757.

35. Hamaoka T, Madewell JE, Podoloff DA, Hortobagyi GN, Ueno NT. Bone imaging in metastatic breast cancer. J Clin Oncol. 2004; 22: 2942-53. doi:10.1200/JCO.2004.08.181.

36. Hatt M, Groheux D, Martineau A, Espie M, Hindie E, Giacchetti S, et al. Comparison between 18F-FDG PET image-derived indices for early prediction of response to neoadjuvant chemotherapy in breast cancer. J Nucl Med. 2013; 54: 341-9. doi:10.2967/jnumed.112.108837.

37. Costelloe CM, Chuang HH, Madewell JE, Ueno NT. Cancer Response Criteria and Bone Metastases: RECIST 1.1, MDA and PERCIST. J Cancer. 2010; 1: 80-92. 
38. Hamaoka T, Costelloe CM, Madewell JE, Liu P, Berry DA, Islam R, et al. Tumour response interpretation with new tumour response criteria vs the World Health Organisation criteria in patients with bone-only metastatic breast cancer. Br J Cancer. 2010; 102: 651-7. doi:10.1038/sj.bjc.6605546.

39. Khokher S, Qureshi MU, Chaudhry NA. Comparison of WHO and RECIST criteria for evaluation of clinical response to chemotherapy in patients with advanced breast cancer. Asian Pac J Cancer Prev. 2012; 13: 3213-8.

40. Peterson LM, Mankoff DA, Lawton T, Yagle K, Schubert EK, Stekhova S, et al. Quantitative imaging of estrogen receptor expression in breast cancer with PET and 18F-fluoroestradiol. J Nucl Med. 2008; 49: 367-74. doi:10.2967/jnumed.107.047506.

41. van Kruchten M, Glaudemans AW, de Vries EF, Beets-Tan RG, Schroder CP, Dierckx RA, et al. PET imaging of estrogen receptors as a diagnostic tool for breast cancer patients presenting with a clinical dilemma. J Nucl Med. 2012; 53: 182-90. doi:10.2967/jnumed.111.092734.

42. Lin NU, Thomssen C, Cardoso F, Cameron D, Cufer T, Fallowfield L, et al. International guidelines for management of metastatic breast cancer (MBC) from the European School of Oncology (ESO)-MBC Task Force: Surveillance, staging, and evaluation of patients with early-stage and metastatic breast cancer. Breast. 2013; 22: 203-10. doi:10.1016/j.breast.2013.03.006.

43. Hayes DF, Bast RC, Desch CE, Fritsche H, Jr., Kemeny NE, Jessup JM, et al. Tumor marker utility grading system: a framework to evaluate clinical utility of tumor markers. J Natl Cancer Inst. 1996; 88: 1456-66.

44. McShane LM, Altman DG, Sauerbrei W, Taube SE, Gion M, Clark GM. REporting recommendations for tumour MARKer prognostic studies (REMARK). Br J Cancer. 2005; 93: 387-91. doi:10.1038/sj.bjc.6602678.

45. Henry NL, Hayes DF. Uses and abuses of tumor markers in the diagnosis, monitoring, and treatment of primary and metastatic breast cancer. Oncologist. 2006; 11: 541-52. doi:10.1634/theoncologist.11-6-541.

46. Mirza NQ, Vlastos G, Meric F, Buchholz TA, Esnaola N, Singletary SE, et al. Predictors of locoregional recurrence among patients with early-stage breast cancer treated with breast-conserving therapy. Ann Surg Oncol. 2002; 9: 256-65.

47. Cristofanilli M. The biological information obtainable from circulating tumor cells. Breast. 2009; 18 Suppl 3: S38-40. doi:10.1016/S0960-9776(09)70270-X.

48. Ntoulia M, Stathopoulou A, Ignatiadis M, Malamos N, Mavroudis D, Georgoulias V, et al. Detection of Mammaglobin A-mRNA-positive circulating tumor cells in peripheral blood of patients with operable breast cancer with $\begin{array}{llll}\text { nested RT-PCR. Clin } & \text { Biochem. 2006; 39: }\end{array}$ doi:10.1016/j.clinbiochem.2006.06.009.

49. Benoy IH, Elst H, Van der Auwera I, Van Laere S, van Dam P, Van Marck E, et al. Real-time RT-PCR correlates with immunocytochemistry for the detection of disseminated epithelial cells in bone marrow aspirates of patients with breast cancer. Br J Cancer. 2004; 91: 1813-20. doi:10.1038/sj.bjc.6602189.

50. Daskalaki A, Agelaki S, Perraki M, Apostolaki S, Xenidis N, Stathopoulos E, et al. Detection of cytokeratin-19 mRNA-positive cells in the peripheral blood and bone marrow of patients with operable breast cancer. Br J Cancer. 2009; 101: 589-97. doi:10.1038/sj.bjc.6605183.

51. Pierga JY, Bidard FC, Denis MG, de Cremoux P. Prognostic value of peripheral blood double detection of CK19 and MUC1 mRNA positive cells detected by RT-quantitative PCR in 94 breast cancer patients with a follow up of 9 years. Mol Oncol. 2007; 1: 267-8. doi:10.1016/j.molonc.2007.09.005.

52. Bidard FC, Mathiot C, Delaloge S, Brain E, Giachetti S, de Cremoux P, et al. Single circulating tumor cell detection and overall survival in nonmetastatic breast cancer. Ann Oncol. 2010; 21: 729-33. doi:10.1093/annonc/mdp391.

53. Pierga JY, Bidard FC, Mathiot C, Brain E, Delaloge S, Giachetti S, et al. Circulating tumor cell detection predicts early metastatic relapse after neoadjuvant chemotherapy in large operable and locally advanced breast cancer in a phase II randomized trial. Clin Cancer Res. 2008; 14: 7004-10. doi:10.1158/1078-0432.CCR-08-0030.

54. Payne RE, Yague E, Slade MJ, Apostolopoulos C, Jiao LR, Ward B, et al. Measurements of EGFR expression on circulating tumor cells are reproducible over time in metastatic breast cancer patients. Pharmacogenomics. 2009; 10: 51-7. doi:10.2217/14622416.10.1.51.

55. Muller V, Riethdorf S, Rack B, Janni W, Fasching PA, Solomayer E, et al Prognostic impact of circulating tumor cells assessed with the CellSearch System and AdnaTest Breast in metastatic breast cancer patients: the DETECT study. Breast Cancer Res. 2012; 14: R118. doi:10.1186/bcr3243.

56. Andreopoulou E, Yang LY, Rangel KM, Reuben JM, Hsu L, Krishnamurthy S, et al. Comparison of assay methods for detection of circulating tumor cells in metastatic breast cancer: AdnaGen AdnaTest BreastCancer Select/Detect versus Veridex CellSearch system. Int J Cancer. 2012; 130: 1590-7. doi:10.1002/ijc.26111.

57. Lennon S, Barton C, Banken L, Gianni L, Marty M, Baselga J, et al. Utility of serum HER2 extracellular domain assessment in clinical decision making: pooled analysis of four trials of trastuzumab in metastatic breast cancer. J Clin Oncol. 2009; 27: 1685-93. doi:10.1200/JCO.2008.16.8351.

58. Kostler WJ, Schwab B, Singer CF, Neumann R, Rucklinger E, Brodowicz T, et al. Monitoring of serum Her-2/neu predicts response and progression-free survival to trastuzumab-based treatment in patients with metastatic breast cancer. Clin Cancer Res. 2004; 10: 1618-24.

59. Tse C, Gauchez AS, Jacot W, Lamy PJ. HER2 shedding and serum HER2 extracellular domain: biology and clinical utility in breast cancer. Cancer Treat Rev. 2012; 38: 133-42. doi:10.1016/j.ctrv.2011.03.008.
60. Dawson SJ, Tsui DW, Murtaza M, Biggs H, Rueda OM, Chin SF, et al. Analysis of circulating tumor DNA to monitor metastatic breast cancer. N Engl J Med. 2013; 368: 1199-209. doi:10.1056/NEJMoa1213261.

61. Zurita M, Lara PC, del Moral R, Torres B, Linares-Fernandez JL, Arrabal SR, et al. Hypermethylated 14-3-3-sigma and ESR1 gene promoters in serum as candidate biomarkers for the diagnosis and treatment efficacy of breast cancer metastasis. BMC Cancer. 2010; 10: 217. doi:10.1186/1471-2407-10-217.

62. Fan J, Deng X, Gallagher JW, Huang H, Huang Y, Wen J, et al. Monitoring the progression of metastatic breast cancer on nanoporous silica chips. Philos Trans A Math Phys Eng Sci. 2012; 370: 2433-47. doi:10.1098/rsta.2011.0444. 